\title{
Laparoscopic Roux-en-Y gastric bypass versus laparoscopic mini gastric bypass in the treatment of obesity: study protocol for a randomized controlled trial
}

Marko Kraljević ${ }^{1 *+}$, Tarik Delko ${ }^{1 \dagger}$, Thomas Köstler², Elena Osto ${ }^{3}$, Thomas Lutz $^{4}$, Sarah Thommen ${ }^{5}$, Raoul A. Droeser ${ }^{1}$, Lincoln Rothwell ${ }^{6}$, Daniel Oertli ${ }^{1}$ and Urs Zingg ${ }^{2}$

\begin{abstract}
Background: Laparoscopic Roux-en-Y gastric bypass (LRYGB) is considered the gold standard in bariatric surgery, achieving durable long-term weight loss with improvement of obesity-related comorbidities. Lately, the laparoscopic mini gastric bypass (LMGB) has gained worldwide popularity with similar results to LRYGB in terms of weight loss and comorbidity resolution. However, there is a lack of randomized controlled trials (RCT) comparing LMGB and LRYGB. This article describes the design and protocol of a randomized controlled trial comparing the outcomes of these two bariatric procedures.

Methods/Design: The trial is designed as a single center, randomized, patient and observer blinded trial. The relevant ethics committee has approved the trial protocol. To demonstrate that LMGB is not inferior to LRYGB in terms of excess weight loss (EWL) the study is conducted as a non-inferiority trial with the sample-size calculations performed accordingly. EWL 12 months after surgery is the primary endpoint, whereas 3-year EWL, morbidity, mortality, remission of obesity related comorbidities, quality of life (QOL) and hormonal and lipid profile changes are secondary endpoints. Eighty patients, 18 years or older and with a body mass index (BMI) between 35 and $50 \mathrm{~kg} / \mathrm{m}^{2}$ who meet the Swiss guidelines for the surgical treatment of morbid obesity will be randomized. The endpoints and baseline measurements will be assessed pre-surgery, peri-surgery and post-surgery (fixed follow up measurements are at discharge and at the time points 6 weeks and 12 and 36 months postoperatively).
\end{abstract}

Discussion: With its 3-year follow up time, this RCT will provide important data on the impact of LMGB and LRYGB on EWL, remission of comorbidities, QOL and hormonal and lipid profile changes.

Trial registration: ClinicalTrials.gov, NCT02601092. Registered on 28 September 2015.

Keywords: Randomized controlled trial, Roux-en-Y gastric bypass, Mini gastric bypass, Outcome, Excess weight loss

\section{Background}

The globally growing incidence of obesity and its related comorbidities is one of the most challenging public health issues [1]. Morbid obesity reduces life expectancy, in particular among younger adults [2]. Bariatric surgery, the only effective treatment for morbidly obese patients,

\footnotetext{
* Correspondence: marko.kraljevic@gmail.com

${ }^{\dagger}$ Equal contributors

'Department of General Surgery, University Hospital Basel, 4031 Basel, Switzerland

Full list of author information is available at the end of the article
}

has shown effective long-term weight loss and significant reduction of obesity-related comorbidities and mortality in randomized controlled trials (RCT) [3-5]. The laparoscopic Roux-en-Y gastric bypass (LRYGB) is considered the gold standard among the different bariatric procedures, achieves durable long-term weight loss and improves obesity-related comorbidities $[6,7]$. The laparoscopic mini gastric bypass (LMGB), first reported by Rutledge et al. [8], has recently gained popularity as a new procedure for the treatment of morbid obesity. LMGB is suggested to be a technically less demanding 
operation compared to LRYGB and offers comparable benefits. Several studies have been published assessing the efficacy and safety of LMGB. The first and only randomized controlled trial comparing the LRYGB to LMGB was carried out by Lee et al. in 2005 [9]. The authors observed excess weight loss (EWL) of $64.9 \%$ after LMGB and of $64.4 \%$ after one and two years, respectively. In comparison, patients with LRYGB had EWL of $58.7 \%$ and $60.0 \%$, respectively. Excess weight (EW) is defined as the amount of weight that is in excess of the ideal body weight (IBW), which is determined as a body mass index (BMI) of $25 \mathrm{~kg} / \mathrm{m}^{2}$. Therefore, EWL is the quotient of weight loss and EW. In addition, LMGB patients had fewer postoperative complications and shorter hospitalization.

Given the increasing number of bariatric procedures performed worldwide [10], the impact on weight loss, morbidity and mortality of LMGB in comparison to LRYGB needs to be further assessed in randomized controlled trials. Therefore, the aim of the current trial is to analyze the EWL and resolution of comorbidities with LMGB and LRYGB. Furthermore, the differences in hormone levels and changes in the lipid status of patients undergoing one of these two operations will be analyzed. These results have the potential to influence bariatric surgery guidelines profoundly and may bring additional insights in the pathophysiology of the intestinal tract after bariatric surgery.

\section{Methods/Design}

\section{Primary objective}

The general objective of this study is to compare LMGB to LRYGB in terms of clinical outcome and safety parameters. The primary objective of the study is to evaluate the non-inferiority of LMGB in comparison to LRYGB in terms of EWL (percent) one year after surgery.

\section{Secondary objective}

The secondary objectives are the long-term effects of LMGB on weight loss, the perioperative and postoperative morbidity and the changes in different pathophysiological parameters during the study period: EWL at 36 months, morbidity, mortality, operative time, length of stay, subjective perception of appetite and satiety, quality of life (QOL), incidence of dumping syndrome, hormone levels (ghrelin, glucagon-like peptide 1, peptide YY, insulin), bile acids and lipid profile and functionality.

\section{Study design and site}

This single center, randomized, controlled, patient and observer blinded, non-inferiority trial is being conducted at the Limmattal Hospital in Zurich-Schlieren, a reference center for bariatric surgery according to the Swiss
Study Group for Morbid Obesity (SMOB). The trial has been registered on ClinicalTrials.gov under the identifier NCT02601092. This protocol has been written in accordance with the Standard Protocol Items: Recommendations for Interventional Trials (SPIRIT) guidelines (Additional file 1). A figure showing the planned visit and examination schedule is presented in Fig. 1.

\section{Sample size}

A total of 80 patients will be randomized, with 40 patients assigned to each treatment arm. A loss of follow up at one year of $2.5 \%$ is considered realistic and it has been calculated to account for a dropout rate of $10 \%$.

\section{Inclusion criteria}

Patients aged 18 years or older with a BMI between 35 and $50 \mathrm{~kg} / \mathrm{m}^{2}$ and eligible for bariatric surgery according to the SMOB guidelines will be enrolled.

\section{Exclusion criteria}

Patients will be excluded on the following basis:

- Missing informed consent

- Lack of conservative weight loss treatment over 2 years

- Malignancy

- Inflammatory bowel disease

- Expected lack of compliance after multidisciplinary evaluation for surgery

- Pregnancy

- BMI over $50 \mathrm{~kg} / \mathrm{m}^{2}$

Preoperatively, patients will be assessed by a nutritionist, endocrinologist, psychiatrist and general surgeon. If more than three appointments are not attended or if there are any contraindications due to severe psychiatric comorbidities, the operation will be postponed or cancelled.

\section{Randomization}

Eligible patients, who give confirmed consent, are randomly assigned to one of the two operating techniques. Randomization will be performed using sequentially numbered, sealed, opaque envelopes. Computergenerated block randomization is used to ensure an equal number of patients in each treatment group. The block size used is not available from the study protocol and is unknown to the investigator, to keep the potential for selection bias at the lowest level possible. Patients will be allocated to a study group by the operating surgeon immediately prior to the operation. 


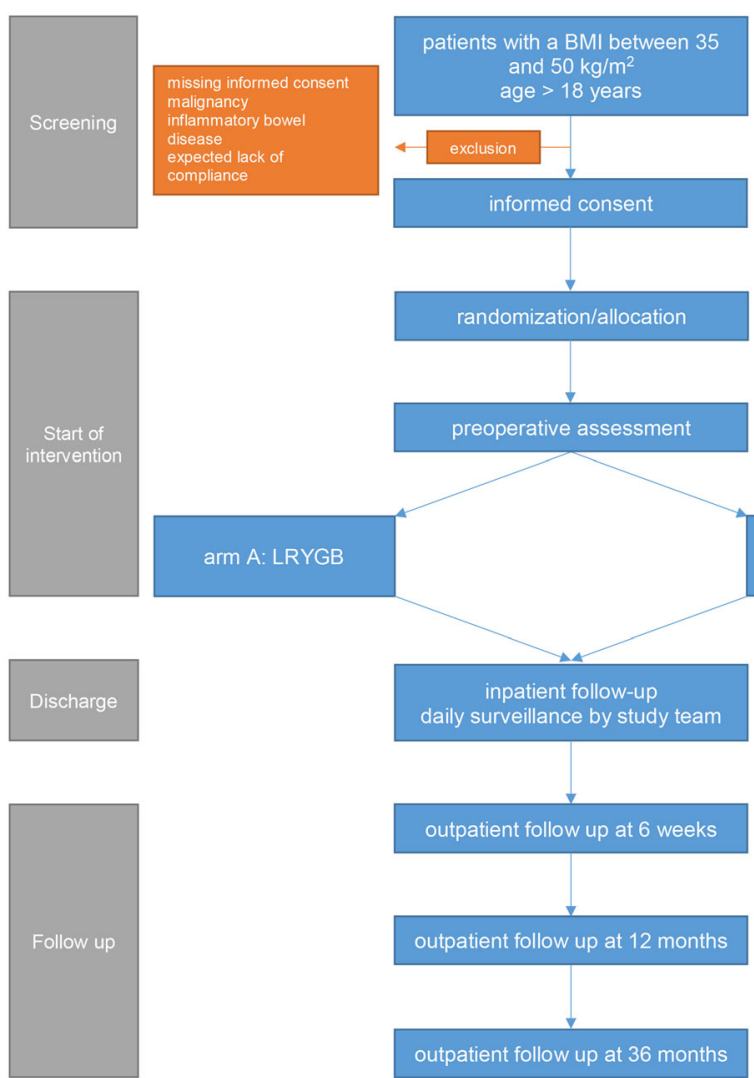

Fig. 1 Schedule of enrollment, interventions, and assessments according to the Standard Protocol Items: Recommendations for Interventional Trials (SPIRIT) guideline. LRYGB laparoscopic Roux-en-Y gastric bypass, LMGB laparoscopic mini gastric bypass, GERD gastroesophageal reflux disease, QOL quality of life

\section{Interventions}

Patients will receive standard preoperative assessment including endocrine, pulmonary function and cardiovascular assessment, psychological assessment, gastroscopy with Helicobacter pylori testing and abdominal ultrasound to check for liver size and gallstones. The operation will always be performed by the same surgeon with experience of performing over 1000 bariatric procedures. Blinded preoperative and postoperative follow up will be independently performed by two study physicians. The study design flow diagram is presented in Fig. 2.

\section{Laparoscopic Roux-en-Y gastric bypass (LRYGB)}

After gastroesophageal junction identification, the stomach is transected with a linear stapler entering the lesser sac $3-5 \mathrm{~cm}$ below the junction, creating a small gastric pouch with a volume of approximately $30 \mathrm{ml}$. A $60-\mathrm{cm}$ biliopancreatic limb is measured and an antecolic endto-side gastroenterostomy is formed using a $25-\mathrm{mm}$ circular stapler. A stapled side-to-side jejunojejunostomy is formed $150 \mathrm{~cm}$ distally (Fig. 3). Intermesenteric and Petersen spaces are closed with non-absorbable sutures. All patients will be discharged on daily multivitamin and calcium tablets. Vitamin levels will be assessed during follow up and supplementation undertaken if required.

\section{Laparoscopic mini gastric bypass (LMGB)}

Stapled division of the stomach at the junction of the body and antrum is undertaken, at a location where a jejunal loop can be comfortably brought up (Fig. 4). A 36 French bougie is passed by the anesthetist and held against the lesser curvature. A very long gastric pouch is created by resection of the stomach parallel to the lesser curvature towards the angle of His. No short gastric vessels are divided. The bypassed stomach lies on the patients left, and the narrow lesser-curvature gastric pouch lies on the patient's midline to the right of the bypassed stomach. A point is selected on the small bowel $200 \mathrm{~cm}$ distal to the ligament of Treitz. The jejunal loop is brought up lying antecolic, and a linear stapler $(60 \mathrm{~mm})$ is used to join the stomach and the small bowel at this point. The linear stapler enterotomy site is then closed with a running suture. The greater omentum is tucked between the gastric tube and the bypassed stomach [8]. Finally, Petersen's space is closed with a non-absorbable suture. All patients will be discharged with daily oral 


\begin{tabular}{|c|c|c|c|c|c|}
\hline \multirow[b]{3}{*}{ TIMEPOINT } & \multicolumn{5}{|c|}{ STUDY PERIOD } \\
\hline & \multirow{2}{*}{$\begin{array}{c}\text { Enrollment } \\
\begin{array}{c}\text { V1 } \\
\text { (preoperative) }\end{array}\end{array}$} & \multirow{2}{*}{$\begin{array}{c}\text { Allocation } \\
\begin{array}{c}\text { V2 } \\
\text { (operation) }\end{array}\end{array}$} & \multicolumn{3}{|c|}{ Post-allocation } \\
\hline & & & $\begin{array}{c}\text { V3 } \\
\text { (6 weeks) }\end{array}$ & $\begin{array}{l}\text { V4 } \\
\text { (1 year) }\end{array}$ & $\begin{array}{c}\text { V5 } \\
\text { (3 years) }\end{array}$ \\
\hline \multirow{4}{*}{$\begin{array}{l}\text { ENROLLMENT: } \\
\text { Eligibility screen } \\
\text { Informed consent } \\
\text { Allocation }\end{array}$} & & & & & \\
\hline & $\mathrm{X}$ & & & & \\
\hline & $\mathrm{X}$ & & & & \\
\hline & & $x$ & & & \\
\hline \multirow{3}{*}{$\begin{array}{c}\text { INTERVENTIONS: } \\
L R Y G B \\
L M G B\end{array}$} & & & & & \\
\hline & & $x$ & & & \\
\hline & & $X$ & & & \\
\hline \multicolumn{6}{|l|}{ ASSESSMENTS: } \\
\hline \multirow{5}{*}{$\begin{array}{c}\text { Weight } \\
\text { Comorbidities } \\
\text { Operation time } \\
\text { Length of hospital } \\
\text { stay } \\
\text { Adverse events }\end{array}$} & $X$ & $X$ & $\mathrm{X}$ & $x$ & $X$ \\
\hline & $X$ & $\mathrm{X}$ & $\mathrm{X}$ & $X$ & $x$ \\
\hline & & $x$ & & & \\
\hline & & $\mathrm{X}$ & & & \\
\hline & & $\mathrm{X}$ & $\mathrm{X}$ & $\mathrm{X}$ & $X$ \\
\hline \multirow{2}{*}{$\begin{array}{l}\text { Hormonal levels } \\
\text { Lipid profile }\end{array}$} & $X$ & & $\mathrm{X}$ & $\mathrm{X}$ & $X$ \\
\hline & $\mathrm{X}$ & & $\mathrm{X}$ & $\mathrm{X}$ & $x$ \\
\hline \multirow{2}{*}{$\begin{array}{c}\text { Bile acids } \\
\text { GERD }\end{array}$} & $X$ & & $x$ & $x$ & $X$ \\
\hline & $X$ & & $x$ & $X$ & $\mathrm{X}$ \\
\hline \multirow{3}{*}{$\begin{array}{l}\text { Satiety degree } \\
\text { Dumping } \\
\text { QOL }\end{array}$} & $\mathrm{X}$ & & $\mathrm{X}$ & $\mathrm{X}$ & $\mathrm{X}$ \\
\hline & $\mathrm{X}$ & & $x$ & $\mathrm{X}$ & $\mathrm{X}$ \\
\hline & $\mathrm{X}$ & & $\mathrm{X}$ & $\mathrm{X}$ & $X$ \\
\hline
\end{tabular}

Fig. 2 Flow diagram of the study design. LMGB laparoscopic mini gastric bypass, LRYGB laparoscopic Roux-en-Y gastric bypass, BMI body mass index

multivitamins and calcium tablets. Vitamin levels will be assessed regularly during follow up and supplementation undertaken if required.

\section{Study visits}

Physicians and study nurses blinded to the intervention will perform study documentation and patient assessment. Since the trial is designed as an observer and patient blinded RCT, information about the surgical procedure will not be disclosed to any assessors during the follow up examinations. There will be five study visits in total. The first visit will be preoperative after informed consent is obtained. Postoperatively, study visits will be performed at discharge, 6 weeks and 12 and 36 months. Each study visit includes collection of data on weight, blood tests, in particular, metabolic parameters such as hormonal assessment, glucose levels and lipid profile. Measurement of metabolic parameters will be conducted preoperatively and 6 weeks and 12 and 36 months postoperatively after a 12-hour fast and at 60 and 120 minutes after a standardized test meal (375 kcal). In addition, questionnaires will be also assessed (Gastrointestinal Quality of Life Index (GIQLI), Simplified Nutritional Appetite Questionnaire (SNAQ), Gastroesophageal Reflux Disease Questionnaire (GERDQ), Bariatric Analysis And Reporting Outcome System (BAROS) and the Sigstad score) at each visit. Furthermore, triglycerides (TG), total cholesterol, high-density lipoprotein (HDL), low-density lipoprotein (LDL), ghrelin, glucagon-like peptide-1 (GLP-1), peptide YY (PYY), glucose, insulin and bile acids will also be evaluated. Postoperatively additional data on morbidity (surgical and non-surgical short-term and longterm complications) and mortality will be collected.

\section{Study endpoints \\ Primary endpoint}

The primary endpoint is the EWL at 12 months after surgery (LRYGB or LMGB). For the EWL calculation (percent) the pre-surgical total body weight (at the patient's hospital entry time) and postsurgical total body weight at one year postoperative is measured.

\section{Secondary endpoints}

- Long-term EWL at 36 months after surgery (in percent)

- Early surgical and non-surgical complications ( $\leq 30$ days) according to the Clavien-Dindo classification [11]

- Operation time

- Length of hospital stay

- Hormonal levels (ghrelin, GLP-1, PYY, glucose and insulin)

- Lipid profile

- Bile acids

- Incidence of GERD

- Satiety degree

- Dumping

- Quality of life questionnaires 


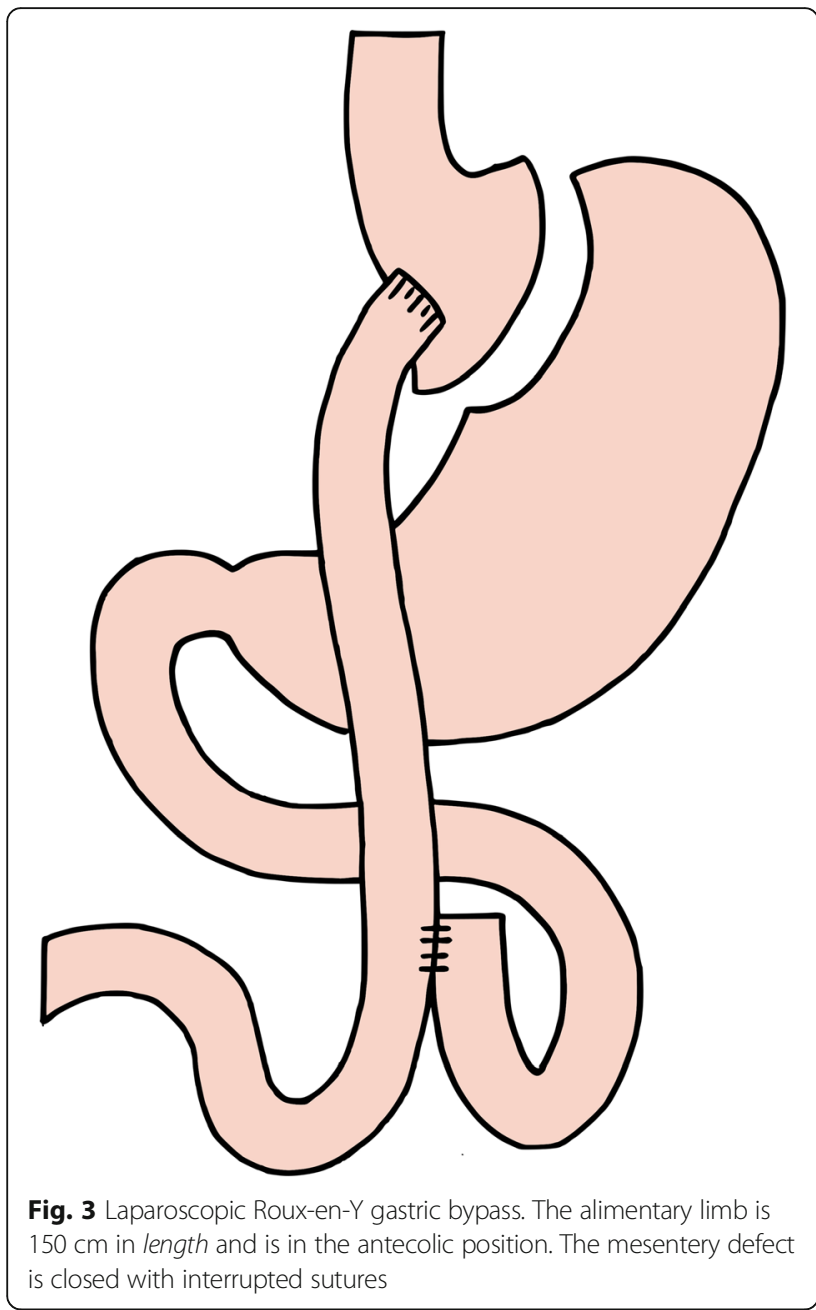

Levels of glucose, insulin and HbA1c will be determined for assessment of glucose homeostasis. TG, total cholesterol, LDL cholesterol (LDL-C), HDL cholesterol (HDL-C) and HDL/LDL cholesterol quotients will be measured to create a lipid profile. Quality of life, satiety degree, incidence of reflux and dumping will be investigated by questionnaires (GIQLI [12], SNAQ [13], GERDQ [14], BAROS [15] and the Sigstad score [16]). Secondary endpoints will be evaluated preoperatively, at discharge, 6 weeks and 12 and 36 months postoperatively.

\section{Blinding}

With the exception of the team in the operating theatre, all medical and non-medical practitioners and patients will be blinded to the procedure. The procedure will be named as study bypass in all medical records. Unblinding is permitted in the case of surgical or medical complications, emergency consultation or other ethical considerations.

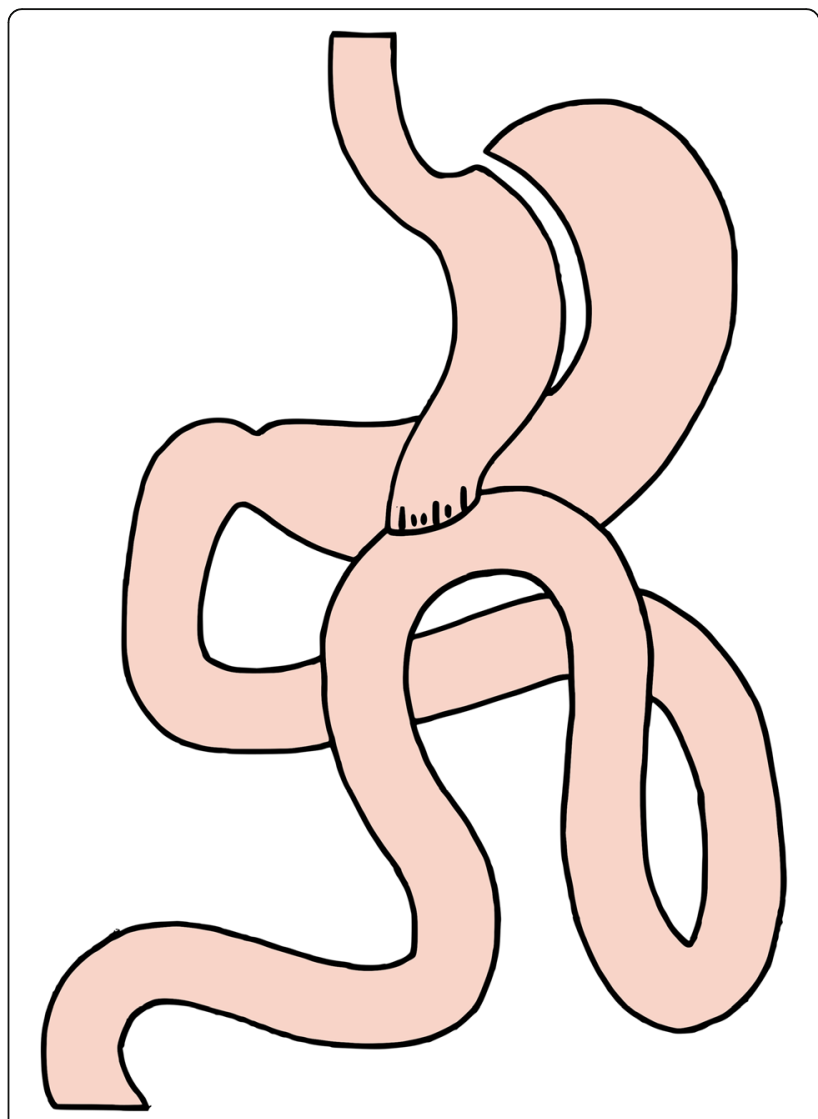

Fig. 4 Laparoscopic mini gastric bypass. The narrow gastric tube is the diameter of a 36 French bougie. The gastroenterostomy is created at the small bowel $200 \mathrm{~cm}$ distal to the Trietz ligament

\section{Statistical analyses}

\section{Sample size}

If LMGB is non-inferior to LRYGB, 72 patients are required (36 patients per study arm) to have an $80 \%$ chance that the lower limit of the one-sided $97.5 \%$ confidence interval (equivalently a 95\% two-sided confidence interval), does not include the non-inferiority limit of $-10 \%$ EWL when assessed at 12 months. Given an anticipated dropout rate of $10 \%$, the total number of patients to be included in the study is 80 (40 patients per arm). The non-inferiority limit of $-10 \%$ EWL was chosen based on expert bariatric opinion. Furthermore a standard deviation of 15 was assumed in both arms according to a study from Lee et al. [9]. For further details on calculations see "Analysis of endpoints".

\section{Analysis of endpoints}

The study results will be reported in adherence to the extension of the Consolidated Standards of Reporting Trials (CONSORT) statement from 2010 on reporting of non-inferiority randomized trials [17]. Summary statistics will be used to describe and compare patient characteristics of all suitable but non-included patients and all 
included patients (overall and stratified for the two treatment arms). Study endpoints will primarily be analyzed for the per protocol (PP) population. Sensitivity analysis will be conducted for the intention-to-treat (ITT) population. Thereby the ITT population includes all randomized patients in the groups to which they were randomly assigned, regardless of their adherence to the entry criteria, regardless of the treatment they actually received and regardless of subsequent withdrawal or deviation from the protocol. In the PP population all protocol violators, including anyone who switched groups or missed measurements are excluded. For each group, number of participants (denominator) included in each analysis and whether the analysis was by original assigned groups will be given. Additional sensitivity analysis will be used if, despite all efforts taken to ensure complete data collection, the number of missing data is non-negligible or could potentially bias the results and conclusions.

\section{Analysis of primary endpoint}

The primary endpoint is the EWL at 12 months postsurgery. To examine non-inferiority of the experimental compared to standard treatment, we assess if the twosided confidence interval of the difference between the mean EWL in the experimental treatment group and the mean EWL in the standard treatment group does not include the non-inferiority limit of $-10 \%$ EWL.

\section{Analysis of secondary endpoints}

To investigate non-inferiority of the experimental treatment in the long term, analysis used for the primary endpoint will be repeated for EWL at 36 months postsurgery. All other endpoints will be analyzed under the assumption of superiority of the experimental treatment compared to the standard treatment. To compare the duration of surgery and the length of hospital stay between the two treatment groups, the Wilcoxon signed-rank test will be conducted (as we assume the data to be right-skewed).

The number of perioperative and postoperative complications will be analyzed by the chi-squared test or Fisher's exact test, dependent on the observed numbers in a $2 \times 2$ contingency table. Additionally, we will compare the risk for complications in the two groups by calculating the risk difference with $95 \%$ confidence interval. The other secondary endpoints will be investigated using appropriate explorative methods and graphical visualization.

\section{Ethical considerations}

Participation in this trial is strictly voluntary and patients are allowed to exit the trial at any point without explanation. All eligible patients are provided an information sheet describing the study with sufficient information for them to make an informed decision about their participation in this study.

The study protocol, patients' information sheets and informed consent forms were approved by the local ethics committee (Kantonale Ethikkommission Zürich, KEK-ZH 2013-0389). In addition, insurance coverage for general liability has been obtained. Patients who decline to participate in this study are treated according to clinical standards. These patients will not be included and no study-specific follow up will be performed.

\section{Participants' confidentiality}

The participants' confidentiality is maintained at all times. For confidentiality reasons, case report forms (CRF) do not contain any personal data on study participants. Members of the ethics committees are obliged to respect confidentiality and to refrain from divulging the participants' identities or any other personal information they might be aware of. Source data in the hospital's electronic patient information systems are secured by personal passwords and handled with respect to medical secrecy.

\section{Archiving and data retention}

The investigator will maintain all study-related records, such as CRFs, medical records, laboratory reports, informed consent documents, safety reports, information regarding participants who discontinued the trial and other pertinent data. All records will be retained by the investigator as long as required by the applicable laws and regulatory requirements (10 years). Thereafter, all data will be destroyed. The study is conducted in compliance with this protocol and according to Good Clinical Practice standards and legal regulations. Serum and plasma samples will be stored in $-80{ }^{\circ} \mathrm{C}$ freezers located in the laboratories where the measurements will be performed, which are the Center for Molecular Cardiology, Institute of Veterinary Physiology at the University of Zurich and the Laboratory of Translational Nutrition Biology at the Federal Institute of Technology (ETH) Zurich.

Freezers are provided with systems for 24-hour monitoring 7 days a week and reporting of temperature control with immediate remote alarm notification and continuous data collection. This provides reliable and durable environmental conditions for temperature requirements of our samples. The serum and plasma samples will be stored for up to 5 years after the completion or termination of the study. After this period, samples will be disposed of as biohazardous waste following policies in force at the University of Zurich and at ETH Zurich for the handling and disposal of biohazardous material. At the completion of the study, there will be a final reconciliation of samples collected, samples used 
and samples remaining. This reconciliation will be reported on a samples accountability form, signed and dated. All stored samples will be coded with a unique storage identifier. The list of patient identifiers is accessible to UZ, TD, MK and EO. The scientists who will carry out analyses on these materials are all experienced researchers. They will not have access to personal identifiers and will not be able to link the results of these tests to personal identifier information. Access to the study results will be restricted. A password system will be utilized to control access. These passwords will be changed on a regular basis. All reports prepared within this study will be prepared such that no individual subject can be identified.

\section{Discussion}

The positive effects of bariatric surgery on weight loss and obesity-related comorbidities and mortality have been widely demonstrated in long-term cohort trials and short-term RCTs $[6,7,18]$. Over time, these procedures have improved in respect to safety and can be offered at a low mortality and morbidity rate $[9,19]$. Various surgical options are available, with LRYGB and laparoscopic sleeve gastrectomy (LSG) being the two most common procedures. In addition, there are many new operations, one of them being the LMGB. The quest for the most effective bariatric procedure is not yet completed as weight loss and improvement of metabolic status, comorbidity and quality of life have only been evaluated in a few randomized controlled trials over a short-term period. This study investigates the effectiveness of LMGB compared to LRYGB, analyzing defined clinical endpoints such as EWL, morbidity and mortality, metabolic changes and quality of life. In addition, the RCT will also answer some important questions about LMGB, such as the course of obesity-related comorbidities and dumping syndrome.

Furthermore, this study addresses the postoperative course of hormones and lipids in these two different bariatric procedures. It has been shown that after bariatric operations the levels of hormones such as GLP-1 and PYY are markedly increased [20]. Surgery improves obesity-associated pro-atherogenic dyslipidemia as well, which is characterized by high LDL and TG and low HDL-C plasma levels. Low HDL-C levels are associated with increased cardiovascular disease risk [21]. It was recently demonstrated that RYGB improved the endothelial protective properties of HDL in obese patients; 12 weeks after RYGB these properties were comparable to those of healthy subjects although the patients were still obese $[22,23]$. Overall, the data indicate that RYGB may achieve a dual HDL benefit, first the rapid restoration of quality to more "healthy" HDL, and second the increase in circulating HDL levels in the longer-term period. The influence of LMGB on changes in lipoprotein profile and function and bile acids are not yet known.

One issue surrounding LMGB that remains highly controversial is the reflux of bile into the gastric pouch and potentially into the esophagus. LMGB is often compared to the refluxogenic loop gastric bypass (LGB) described by Mason et al. [24], which consists of a large gastric pouch transected horizontally with the gastrojejunostomy close to the gastroesophageal junction. However, LMGB consists of a long narrow vertical gastric pouch, thus, reflux into the esophagus is not believed to be an LMGB-specific problem. By contrast, the surgical technique of LMBG is comparable to the Billroth II procedure performed in peptic ulcer disease and gastric cancer. In LMBG and Billroth II, the most important parallel is the single anastomosis bypass of the duodenum without a Roux-limb. The LMGB distance of the gastrojejunostomy to the esophagus is longer than in Billroth II. Furthermore, the biliopancreatic limb in LMGB measures $200 \mathrm{~cm}$ compared to $60-80 \mathrm{~cm}$ for the Billroth II operation.

In conclusion, the perfect bariatric procedure is technically easy and safe, and leads to adequate EWL and improvement in obesity-related comorbidities. In contrast to the widely used LRYGB and LSG, the LMGB might fulfill all of these criteria. The current study will answer questions about safety, effectiveness and pathophysiological changes in obesity-relevant hormones and lipids after LMGB. These findings might therefore influence decision-making in bariatric surgery implementing current surgical protocols towards personalized indications.

\section{Trial status}

The trial has received ethics approval, and enrollment in the trial has begun but has not yet reached full enrollment.

\section{Additional file}

Additional file 1: SPIRIT checklist of included items in our clinical trial protocol (DOCX $64 \mathrm{~kb})$

\section{Abbreviations}

BAROS: Bariatric Analysis and Reporting Outcome System; BMI: Body mass index; CRF: Case report form; EGD: Esophagogastroduodenoscopy;

EW: Excess weight; EWL: Excess weight loss; GERDQ: Gastroesophageal Reflux Disease Questionnaire; GIQLI: Gastrointestinal Quality of Life Index; GLP-

1: Glucagon-like peptide 1; HDL: High density lipoprotein; IBW: Ideal body weight; ITT: Intention-to-treat; LDL: Low density lipoprotein;

LMGB: Laparoscopic mini gastric bypass; LRYGB: Laparoscopic Roux-en-Y gastric bypass; PP: Per protocol; PYY: Peptide YY; QOL: Quality of life; RCT: Randomized controlled trial; RYGB: Roux-en-Y gastric bypass: SMOB: Swiss Study Group for Morbid Obesity; SNAQ: Simplified Nutritional Appetite Questionnaire; SPIRIT: Standard Protocol Items: Recommendations for Interventional Trials; TG: Triglycerides 


\section{Acknowledgements}

The authors would like to thank all support personnel for their hard work and dedication.

\section{Funding}

We have not received external funding for the trial. There are no commercial sponsors.

\section{Availability of data and materials}

All data will be available for other research groups interested in conduction systematic reviews. Requests for data should be directed to the Primary Investigator, Urs Zingg, urs.zingg@spital-limmattal.ch.

\section{Authors' contributions}

TD is responsible for the setting up and coordination of the study. He drafted this manuscript. He has revised this manuscript critically for important intellectual content and given final approval of this final version. MK is responsible for data acquisition and follow up and he drafted this manuscript. He has revised this manuscript critically for important intellectual content and given final approval of this final version. He agrees to be accountable for all aspects of the work in ensuring that questions related to the accuracy or integrity of any part of the work are appropriately investigated and resolved. TK is the operating surgeon and responsible for the recruitment of patients. He participated in the conception and design of the study. He has revised this manuscript critically for important intellectual content and given final approval of this final version. He agrees to be accountable for all aspects of the work in ensuring that questions related to the accuracy or integrity of any part of the work are appropriately investigated and resolved. EO is part of the data acquisition team and will be responsible for the assessment of hormonal and lipid evaluation. She has revised this manuscript critically for important intellectual content and given final approval of this final version. She agrees to be accountable for all aspects of the work in ensuring that questions related to the accuracy or integrity of any part of the work are appropriately investigated and resolved. TL is part of the data acquisition team and will be responsible for the assessment of hormonal and lipid evaluation. He has revised this manuscript critically for important intellectual content and given final approval of this final version. He agrees to be accountable for all aspects of the work in ensuring that questions related to the accuracy or integrity of any part of the work are appropriately investigated and resolved. ST participated in drafting this manuscript. She will be responsible for trial statistical analysis. She has revised this manuscript critically for important intellectual content and given final approval of this final version. RD is responsible for data acquisition. He has revised this manuscript critically for important intellectual content and given final approval of this final version. He agrees to be accountable for all aspects of the work in ensuring that questions related to the accuracy or integrity of any part of the work are appropriately investigated and resolved. LR has revised this manuscript critically for important intellectual content and given final approval of this final version. He agrees to be accountable for all aspects of the work in ensuring that questions related to the accuracy or integrity of any part of the work are appropriately investigated and resolved. DO is responsible for data acquisition. He has revised this manuscript critically for important intellectual content and given final approval of this final version. He agrees to be accountable for all aspects of the work in ensuring that questions related to the accuracy or integrity of any part of the work are appropriately investigated and resolved. UZ initiated, designed and conceived this study. He is the principal investigator and responsible for overall study coordination. He has revised this manuscript critically for important intellectual content and given final approval of this final version. He agrees to be accountable for all aspects of the work in ensuring that questions related to the accuracy or integrity of any part of the work are appropriately investigated and resolved. All authors read and approved the final manuscript.

\section{Authors' information}

Not applicable.

\section{Competing interests}

The authors declare that they have no competing interests.

\section{Consent for publication}

Not applicable.

\section{Ethics approval and consent to participate}

The study protocol, patients' information sheets and informed consent forms were approved by the local ethics committee (Kantonale Ethikkommission Zürich, KEK-ZH 2013-0389). The study was also registered with the ClinicalTrials.gov registry (Registration number NCT02601092). Written informed consent will be obtained and documented for all study participants.

\section{Publisher's Note}

Springer Nature remains neutral with regard to jurisdictional claims in published maps and institutional affiliations.

\section{Author details}

'Department of General Surgery, University Hospital Basel, 4031 Basel, Switzerland. ${ }^{2}$ Department of General Surgery, Limmattal Hospital, 8952 Zurich-Schlieren, Switzerland. ${ }^{3}$ FNH Laboratory of Translational Nutrition Biology, ETH Zurich, 8603 Schwerzenbach, Switzerland. Institute of Veterinary Physiology, Vetsuisse Faculty and Centre of Integrative Human Physiology, University of Zurich, 8057 Zurich, Switzerland. ${ }^{5}$ Basel Institute for Clinical Epidemiology and Biostatistics, University Hospital Basel, 4031 Basel, Switzerland. ${ }^{6}$ Department of General Surgery, Ipswich General Hospital, Ipswich, Queensland 4305, Australia.

Received: 9 November 2016 Accepted: 27 April 2017

Published online: 22 May 2017

\section{References}

1. Danaei G, Finucane MM, Lu Y, Singh GM, Cowan MJ, Paciorek CJ, et al. National, regional, and global trends in fasting plasma glucose and diabetes prevalence since 1980: systematic analysis of health examination surveys and epidemiological studies with 370 country-years and 2.7 million participants. Lancet. 2011;378:31-40.

2. Fontaine KR, Redden DT, Wang C, Westfall AO, Allison DB. Years of life lost due to obesity. J Am Med Assoc. 2003;289:187-93.

3. Sjöström L, Narbro K, Sjöström CD, Karason K, Larsson B, Wedel H, et al. Effects of bariatric surgery on mortality in Swedish obese subjects. N Engl J Med. 2007:357:741-52.

4. Adams T, Gress R, Simper Smith S, Halverson R, Simper Smith S, Rosamond W, et al. Long-term mortality after gastric bypass surgery. N Engl J Med. 2007;357:753-61.

5. Colquitt J, Pickett K, Loveman E, Frampton G. Surgery for weight loss in adults. Cochrane Database Syst Rev. 2014;8. https:/www.ncbi.nlm.nih.gov/ pubmed/?term =Colquitt+J\%2C+Pickett+K\%2C+Loveman+E\%2C+Frampton +G.+Surgery+for+weight+loss+in+adults.+Cochrane+Database+Syst+Rev,

6. Schauer PR, Ikramuddin S, Gourash W, Ramanathan R, Luketich J. Outcomes after laparoscopic Roux-en-Y gastric bypass for morbid obesity. Ann Surg. 2000;232:515-29.

7. Schauer PR, Bhatt DL, Kirwan JP, Wolski K, Brethauer SA, Navaneethan SD, et al. Bariatric surgery versus intensive medical therapy for diabetes - 3-year outcomes. N Engl J Med. 2014;370(21):2002-13.

8. Rutledge R. The mini-gastric bypass: experience with the first 1274 cases. Obes Surg. 2014;370(21):2002-13.

9. Lee W-J, Yu P-J, Wang W, Chen T-C, Wei P-L, Huang M-T. Laparoscopic Roux-en-Y versus mini-gastric bypass for the treatment of morbid obesity. Ann Surg. 2005;242:20-8.

10. Buchwald H, Oien DM. Metabolic/bariatric surgery worldwide 2008. Obes Surg. 2009;19:1605-11.

11. Clavien PA, Barkun J, de Oliveira ML, Vauthey JN, Dindo D, Schulick RD, et al. The Clavien-Dindo classification of surgical complications. Ann Surg. 2009; 250:187-96

12. Eypasch E, Williams J, Wood-Dauphinee S, Ure BM, Schmülling C,

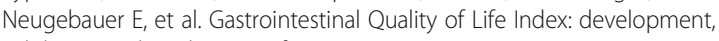
validation and application of a new instrument. Br J Surg. 1995;82:216-22.

13. Wilson M, Thomas D, Rubenstein L, Chibnall J, Anderson S, Baxi A, et al. Appetite assessment: simple appetite questionnaire predicts weight loss in community-dwelling adults and nursing home residents. Am J Clin Nutr. 2005;82:1074-81.

14. Jones R, Junghard O, Dent J, Vakil N, Halling K, Wernersson B, et al. Development of the GerdQ, a tool for the diagnosis and management of gastro-oesophageal reflux disease in primary care. Aliment Pharmacol Ther. 2009;30:1030-8. 
15. Oria EH, Moorehead K. Bariatric Analysis and Reporting System (BAROS). Obes Surg. 1998;8:487-99.

16. Sigstad H. A clinical diagnostic index in the diagnosis of the dumping syndrome. Changes in plasma volume and blood sugar after a test meal. Acta Med Scand. 1970;188:479-86.

17. Piaggio G, Elbourne D, Pocock S, Evans S, Altman D. Reporting of noninferiority and equivalence randomized trials: extension of the CONSORT 2010. JAMA. 2012;308:2594-604.

18. Paulus GF, de Vaan LEG, Verdam FJ, Bouvy ND, Ambergen TAW, van Heurn LWE. Bariatric surgery in morbidly obese adolescents: a systematic review and meta-analysis. Obes Surg. 2015;860-78.

19. Peterli R, Borbély Y, Kern B, Gass M, Peters T, Thurnheer M, et al. Early results of the Swiss Multicentre Bypass or Sleeve Study (SM-BOSS): a prospective randomized trial comparing laparoscopic sleeve gastrectomy and RouX-en-Y gastric bypass. Ann Surg. 2013;258:690-5.

20. Peterli R, Steinert RE, Woelnerhanssen B, Peters T, Christoffel-Courtin C, Gass M, et al. Metabolic and hormonal changes after laparoscopic Roux-en-Y gastric bypass and sleeve gastrectomy: a randomized, prospective trial. Obes Surg. 2012;22:740-8.

21. Abbott RD, Wilson PW, Kannel WB, Castelli WP. High density lipoprotein cholesterol, total cholesterol screening, and myocardial infarction. The Framingham Study. Arteriosclerosis. 1998;8:207-11.

22. Aminian A, Zelisko A, Kirwan JP, Brethauer SA, Schauer PR. Exploring the impact of bariatric surgery on high density lipoprotein. Surg Obes Relat Dis. 2015;11:238-47.

23. Osto E, Doytcheva P, Corteville C, Bueter M, Dörig C, Stivala S, et al. Rapid and body weight-independent improvement of endothelial and highdensity lipoprotein function after Roux-en-Y Gastric bypass: role of glucagon-like peptide-1. Circulation. 2015;131:871-81.

24. Mason E, Ito C. Gastric bypass in obesity. Surg Clin North Am. 1967;47:1345-52.

\section{Submit your next manuscript to BioMed Central and we will help you at every step:}

- We accept pre-submission inquiries

- Our selector tool helps you to find the most relevant journal

- We provide round the clock customer support

- Convenient online submission

- Thorough peer review

- Inclusion in PubMed and all major indexing services

- Maximum visibility for your research

Submit your manuscript at www.biomedcentral.com/submit

C) Biomed Central 